\title{
Parent-provider paediatric literacy communication: A curriculum for future primary care providers
}

\author{
Tiffany Kindratt ${ }^{1} \cdot$ Brittany Bernard $^{2}$. Jade Webb ${ }^{3} \cdot$ Patti Pagels $^{4}$
}

Published online: 25 March 2019

(c) The Author(s) 2019

\begin{abstract}
Background Reach Out and Read promotes early literacy and school readiness by incorporating book delivery and anticipatory guidance into well-child visits. There is a need to train future healthcare providers in the knowledge and skills to communicate with parents/caregivers about early childhood literacy. We developed and evaluated a curriculum to improve learners' knowledge, attitudes, and skills towards the incorporation of parent-provider literacy communication into well-child visits.

Methods Family medicine residents $(n=30)$, physician assistant students $(n=36)$, and medical students $(n=28)$ participated in a curriculum consisting of service learning, online didactic training, objective structured clinical exams (OSCEs) and a debriefing session. Standardized patients (SPs; 6 months to 5 years) and standardized patient caregivers were recruited and trained. Learners were evaluated on their abilities to offer books to patients, provide anticipatory guidance, and demonstrate parent-provider communication skills. Knowledge, attitudes, and satisfaction were collected pre- and post-curriculum.

Results Significant increases in total knowledge were observed after completing curriculum activities $(p<0.001)$. All attitudes improved after training $(p<0.05)$. All learners $(100 \%)$ recommended that caregivers talk back and forth with their 6- to 12-month-old babies and make eye contact. Few (18.2\%) learners recommended playing games like 'peek-a-boo' while reading. When caregivers evaluated learners' basic parent-provider communication skills, all reported that the learners treated them with respect and used plain language.

Discussion Our curriculum extends beyond previous studies by measuring recommended books, anticipatory guidance, and communication skills using paediatric SPs and standardized patient caregivers. Curriculum activities can be tailored to best promote parent-provider literacy communication training in other programs.
\end{abstract}

Keywords Reach Out and Read · Objective structured clinical exam · Primary care · Early childhood literacy · Physician assistant $\cdot$ Postgraduate $\cdot$ Medical students $\cdot$ Paediatrics

\section{Background}

Electronic supplementary material The online version of this article (https://doi.org/10.1007/s40037-019-0503-8) contains supplementary material, which is available to authorized users.

Tiffany Kindratt

Tiffany.Kindratt@utsouthwestern.edu

1 Department of Physician Assistant Studies, University of Texas Southwestern Medical Center, School of Health Professions, Dallas, TX, USA

2 Children's Minnesota, Minneapolis, MN, USA

3 Questcare Partners, Dallas, TX, USA

4 Community Health Section, Department of Family and Community Medicine, University of Texas Southwestern Medical School, Dallas, TX, USA
Efforts have been made to improve patient-provider communication education in medical schools, residencies, and physician assistant programs [1]. Accrediting bodies require instruction in communication skills for an effective exchange of health information with patients $[2,3]$. Limited training has focused on communication with parents/ caregivers. The American Academy of Family Physicians recommends that the curriculum be designed to train future providers how to communicate with patients and caregivers and promote early literacy development at well-child visits [4].

Since 1989, Reach Out and Read has promoted early literacy in clinics in the United States through shared reading between parents and children. Evidence suggests that shared reading improves brain function, language, and 
school readiness [5]. Reach Out and Read promotes shared reading by distributing books to patients aged 6 months to 5 years, delivering anticipatory guidance to parents on reading aloud, and assessing developmental literacy milestones during well-child visits [6, 7]. A recent study demonstrated that by including Reach Out and Read in clinics, children were more receptive to reading, more likely to read with family and, ultimately, had improved language skills [8]. While the literature has expanded on the benefits of Reach Out and Read, limited research has focused on training providers. Studies have shown that residency training may improve knowledge, skills, and attitudes towards paediatric literacy concepts $[9,10]$. However, these studies lacked simulated experiences where learners could practice communication skills with patients/caregivers and receive formative feedback. Objective structured clinical exams (OSCEs) have been used for over 40 years to provide formative feedback [11]. OSCEs utilize brief (5-10 min) clinical encounters with trained standardized patients (SPs). While OSCEs were developed for assessments of history and physical examinations, their use has expanded with the changing healthcare landscape. OSCEs are now utilized to measure patient-provider communication among patients from special populations (e.g. limited health literacy) [12], and to incorporate health information technology (e.g. mobile phone applications) [13] into patient encounters. There remains a gap in the literature on the use of OSCEs for training future providers how to communicate with paediatric patients and parents/caregivers. No studies have evaluated paediatric literacy training for physician assistant students, medical students, or interprofessional learners.

Our purpose was to develop and evaluate a curriculum to improve learners' knowledge, attitudes, and skills towards paediatric literacy concepts. In this article, we present our findings and curriculum materials for use by other training programs.

\section{Evaluation of innovation}

\section{Methods}

Learners (family medicine residents $n=30$; medical students $n=28$; physician assistant students $n=36$ ) participated from the University of Texas Southwestern Medical Center. Learners completed all or some curriculum activities as part of a community-based rotation (residents) [14], preclinical elective (medical students), or population healthrelated course (physician assistant students).

\section{Development and implementation}

The curriculum included service learning, online didactic training, OSCE stations, and a classroom-based debriefing session [15] developed from previous research and curriculum recommendations $[4,9,10,16,17]$. Learners had opportunities for service learning at clinics embedded in local homeless shelters. Reach Out and Read programs began at the shelter for women and children in 2013 [17] and the domestic violence shelter in 2016. The shelter for women and children has a student-run clinic managed by medical and physician assistant students. Each session is staffed with four learners and one manager. Learners are supervised by the medical director, who is a certified physician assistant. In 2015, managers received lecture-based training on how to distribute books, discuss anticipatory guidance with parents, and document both in electronic medical records (EMRs). This training improved managers' skills [17]. However, other volunteer learners did not receive training. Based on the growing number of volunteers and lack of training available, there was a need to implement a comprehensive parent-provider paediatric literacy curriculum. The training benefits patients and caregivers by equipping learners with knowledge and skills to provide anticipatory guidance, discuss reading, and provide books at no cost to homeless families.

The didactic curriculum was developed to expand the national Reach Out and Read training [16]. The online training included four modules: (1) benefits of Reach Out and Read; (2) ways to incorporate books in children's lives; (3) teachable moments/techniques for clinical settings; and (4) research. The expanded training was developed so learners could: complete the training without being listed as an official provider for a designated Reach Out and Read clinic; obtain and complete trainings without internet access; and learn how to document book delivery and anticipatory guidance in EMRs.

OSCEs were developed and implemented based on their acceptability in our programs $[12,13]$ and ability to improve communication skills [11]. Paediatric standardized patients (SPs) and standardized patient caregivers were recruited and trained. Caregivers received training materials 2 weeks beforehand by email and 1 hour of in-person training prior to administering stations. Stations represented children accompanied by a mother, father, grandparent, or non-relative caregiver. Learners had 2 minutes to read a prompt on the door of each station instructing him/her to: give a book to the patient; provide anticipatory guidance; and practise basic parent-provider communication. Each learner completed two stations: (1) infants/toddlers $(<2$ years old $)$ and (2) pre-schoolers (2-5 years old). OSCE station overviews, prompts, and caregivers scripts are provided in the online Supplementary Tables $1-5$. 
Table 1 Demographics, knowledge ${ }^{\mathrm{a}}$, attitudes ${ }^{\mathrm{a}}$, and satisfaction of medical learners

\begin{tabular}{|c|c|c|c|}
\hline Demographics $(n=94)$ & $N(\%)$ & & \\
\hline \multicolumn{4}{|l|}{ Gender } \\
\hline Female & $65(69.1)$ & & \\
\hline Male & $29(30.9)$ & & \\
\hline \multicolumn{4}{|l|}{ Race/Ethnicity } \\
\hline White/Caucasian & $30(31.9)$ & & \\
\hline Black/African American & $3(3.2)$ & & \\
\hline Hispanic & $11(11.7)$ & & \\
\hline Asian American & $25(26.6)$ & & \\
\hline Other/not reported & $25(26.6)$ & & \\
\hline \multicolumn{4}{|l|}{ Medical learner group } \\
\hline Physician assistant students & $36(38.3)$ & & \\
\hline Medical students & $28(29.8)$ & & \\
\hline Family medicine residents & $30(31.9)$ & & \\
\hline Pre- and Post-Test comparisons & $\begin{array}{l}\text { Pre-test } \\
\text { correct }\end{array}$ & $\begin{array}{l}\text { Post-test } \\
\text { correct }\end{array}$ & \\
\hline$(\mathbf{n}=53)$ & n $(\%)$ & n $(\%)$ & $\mathbf{P}$ \\
\hline \multicolumn{4}{|l|}{ Knowledge $e^{b}$} \\
\hline 1. Reach Out and Read serves over 5 million children annually in the US & $50(94.3)$ & $46(95.8)$ & 0.0833 \\
\hline 2. Watching Sesame Street is least likely to foster reading and writing & $30(56.6)$ & $39(81.3)$ & 0.0075 \\
\hline 3. Most children turn pages in board books by 18 months & $48(90.6)$ & $45(93.8)$ & 0.4142 \\
\hline 4. It is important to read a book word for word, even if very young & $36(67.9)$ & $43(89.6)$ & 0.0075 \\
\hline \multirow{2}{*}{$\begin{array}{l}\text { 5. Reach Out and Read books should be given to all children aged } 12 \text { and } \\
\text { under (answer: false-under } 5 \text { years) }\end{array}$} & $6(11.3)$ & $14(29.2)$ & 0.1655 \\
\hline & Mean (SD) & Mean (SD) & \\
\hline Total knowledge & $3.21(0.93)$ & $3.90(0.69)$ & 0.0002 \\
\hline \multicolumn{4}{|l|}{ Attitudes ${ }^{c}$} \\
\hline 6. I feel comfortable assessing literacy during paediatric clinic visits & $2.83(0.81)$ & $3.89(0.52)$ & $<0.0001$ \\
\hline 7. Parents are (not) offended by questions about literacy & $3.15(0.96)$ & $3.74(0.90)$ & 0.0054 \\
\hline 8. The clinic is an appropriate place to encourage literacy & $4.12(0.81)$ & $4.57(0.54)$ & 0.0034 \\
\hline $\begin{array}{l}\text { 9. Literacy assessments and related anticipatory guidance tips are (not) only } \\
\text { necessary when children are close to school age }\end{array}$ & $2.90(1.35)$ & $4.38(1.01)$ & $<0.0001$ \\
\hline Post-curriculum satisfaction & - & Mean (SD) & - \\
\hline 10. Clear objectives were provided & - & $3.89(0.73)$ & - \\
\hline 11. Information was provided that met my training needs & - & $3.98(0.68)$ & - \\
\hline 12. I can use this information to improve patient care & - & $4.30(0.63)$ & - \\
\hline 13. Overall, the training was very good & - & $4.16(0.67)$ & - \\
\hline
\end{tabular}

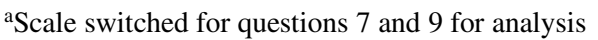

${ }^{\mathrm{b}} \mathrm{McNemar}$ tests used for comparisons, significance level $\mathrm{p}<0.05$

${ }^{c}$ Wilcoxon signed-rank tests used for comparisons, significance level $\mathrm{p}<0.05$

Learners reflected on their experiences with a certified physician assistant and the director of Reach Out and Read Texas during the debriefing session immediately following the OSCE stations. Learners shared service learning experiences and discussed how they would incorporate the knowledge and skills they learned into their future practice.

\section{Evaluations}

Demographics were collected (gender, race/ethnicity) from program data sources. Outcomes were evaluated using three of Kirkpatrick's levels of evaluation including (1) reaction, (2) learning, and (3) behaviour [18]. Learners received emails with a link to pre- and post-test surveys. Pre-test data were collected at the beginning of each course/rotation. Post-test data were collected immediately after completing the OSCE and debriefing session. Among those who did not complete the OSCE and debriefing session, post-tests were collected immediately after online training.

Learners answered four questions assessing their satisfaction with the curriculum activities. To measure changes in self-perceived knowledge and attitudes, learners com- 
pleted nine pre- and post-test items using the same questions (see Tab. 1 for questions). Knowledge was measured with dichotomous variables (correct vs. incorrect). All preand post-knowledge questions were added together to create total pre- and post-knowledge scores. Attitudes were measured using 5-point Likert scales $(1=$ strongly disagree; $5=$ strongly agree).

To measure behaviour, caregivers scored learners (yes or no) on their ability to provide anticipatory guidance (e.g. name things), recommend age-appropriate books (e.g. board books), and demonstrate communication skills (e.g. introduced him/herself). OSCE scoresheets were developed based on Reach Out and Read's 'Milestones of Early Literacy Development Chart' [16]. See online Supplemental Tables $1-5$ for questions.

\section{Analysis}

Data analysis was performed using STATA 14.0. Means and standard deviations were used to report satisfaction. McNemar tests were used to determine significant differences in pre- and post-test knowledge items. Wilcoxon signedrank tests were used to determine significant differences between pre- and post-knowledge total scores. Wilcoxon tests were used to determine significant differences between pre- and post-attitudes. Frequencies and percentages were used to describe learners who demonstrated the expected behaviours during OSCE stations. Wilcoxon and chi-square tests were used to compare changes in attitudes, knowledge, and post-satisfaction between learners who did and did not participate in the OSCE and debriefing session.

The University of Texas Southwestern Medical Center's Institutional Review Board deemed this project as non-human subjects research.

\section{Results}

Tab. 1 reports demographics, and pre-, and post-test comparisons. Most learners were female (69\%). Over half were white $(32 \%)$ or Asian $(27 \%)$ race/ethnicity. More were physician assistant students $(38 \%)$ compared with residents $(32 \%)$ or medical students $(30 \%)$. All learners were required to complete the online training. There were 68 learners who gained service learning experiences at the homeless shelters, 53 who completed the pre- and post-tests, and 38 who completed the OSCE stations and debriefing session. Only two residents and no medical students were able to participate in the OSCEs and debriefing session.

Learners gained the most knowledge on the impact of technology and reading aloud on language development. Most $(81 \%)$ learners identified that watching television shows like 'Sesame Street' was least likely to foster read- ing and writing after training compared with only $57 \%$ before training $(p=0.0075)$. Total knowledge scores were significantly higher post-curriculum $(p<0.0001)$. All attitudes improved after training. Learners were more likely to disagree with the statement 'literacy assessments and related anticipatory guidance tips are only necessary when children are close to school age' $(p<0.0001)$. Learners expressed their greatest level of satisfaction with their ability to use what they learned to improve patient care $($ mean $=4.30)$. No significant differences were found in knowledge, attitudes, or post-curriculum satisfaction between learners who did and did not participate in the OSCE stations and debriefing session.

Tab. 2 reports frequencies and percentages of learners who demonstrated the expected behaviour during OSCE stations. For 6- to 12-month-old SPs, all learners (100\%) recommended that caregivers talk back and forth with their babies and make eye contact. Few (18.2\%) recommended playing games like 'peek-a-boo' while reading. Most $(92.6 \%=12-24$ months; $90.9 \%=2-3$ years $)$ recommended caregivers use books in daily routines. When caregivers evaluated learners' basic parent-provider communication skills, most learners introduced themselves when they entered the room $(98.6 \%)$ and sat while talking to the SP and caregiver $(92.1 \%)$.

\section{Reflection and critical review}

Our purpose was to develop and evaluate an innovative curriculum to improve interprofessional learners' knowledge, attitudes, and skills in paediatric literacy concepts. We found significant improvements in knowledge and attitudes after completion of curriculum activities, which is similar to previous studies with residents [8, 9]. Our study extends beyond this research by incorporating interprofessional learners and using paediatric SPs and standardized patient caregivers. All learners demonstrated at least one basic communication skill during OSCE stations. These results are similar to previous research evaluating communication skills using OSCEs with adult patients in our program [13]. Although there were no significant differences in results among learners who did and did not participate in OSCE stations, this innovative component allows learners to gain first-hand, simulated experiences with paediatric patients and parents, which is often neglected in medical education. Most medical and physician assistant students had no prior experience in practising parent-provider communication.

Our study contributes to the literature by highlighting the importance of providers discussing shared reading and early childhood literacy with parents during well-child visits. Recent research has emphasized how Reach Out and 


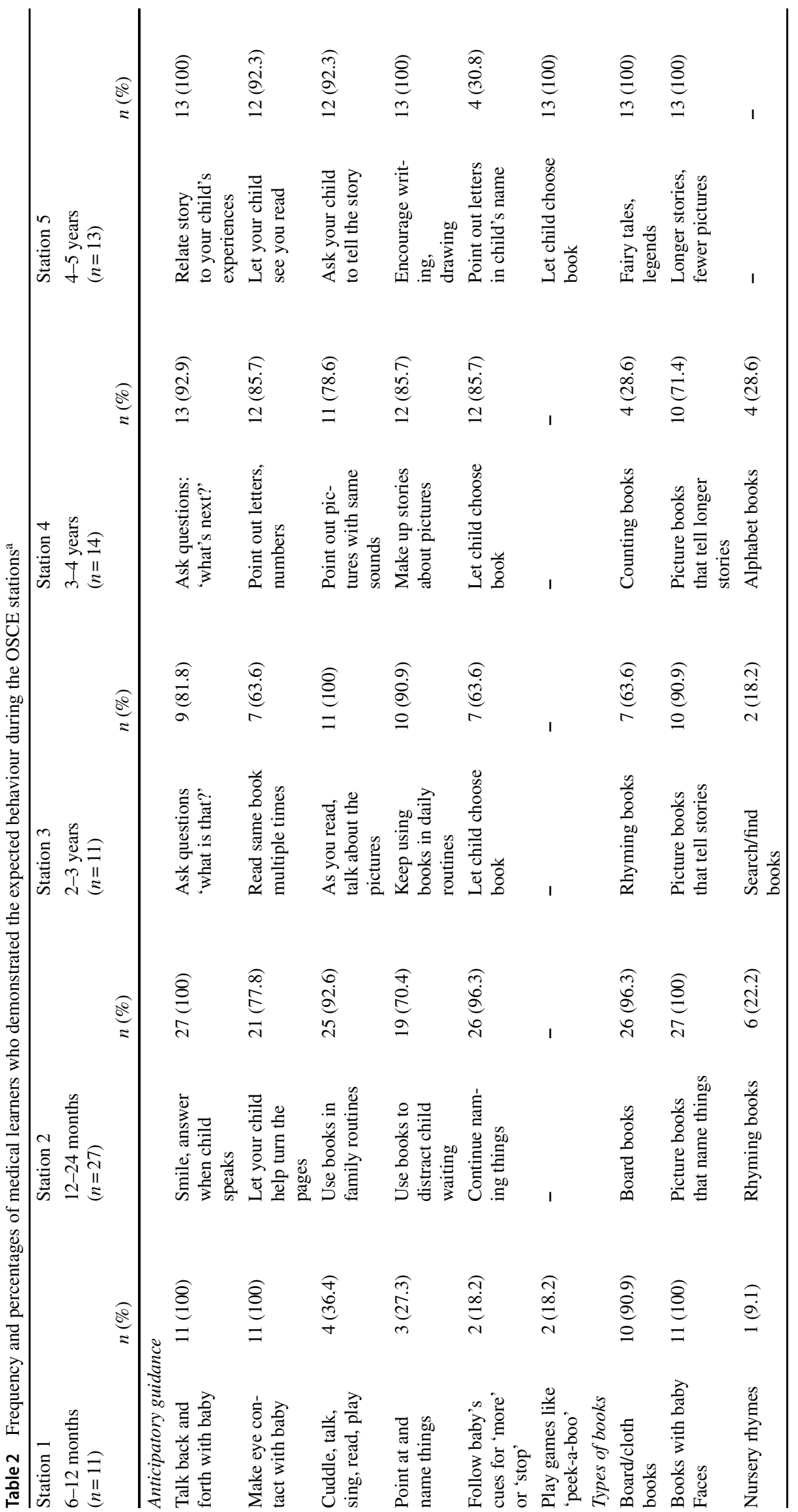




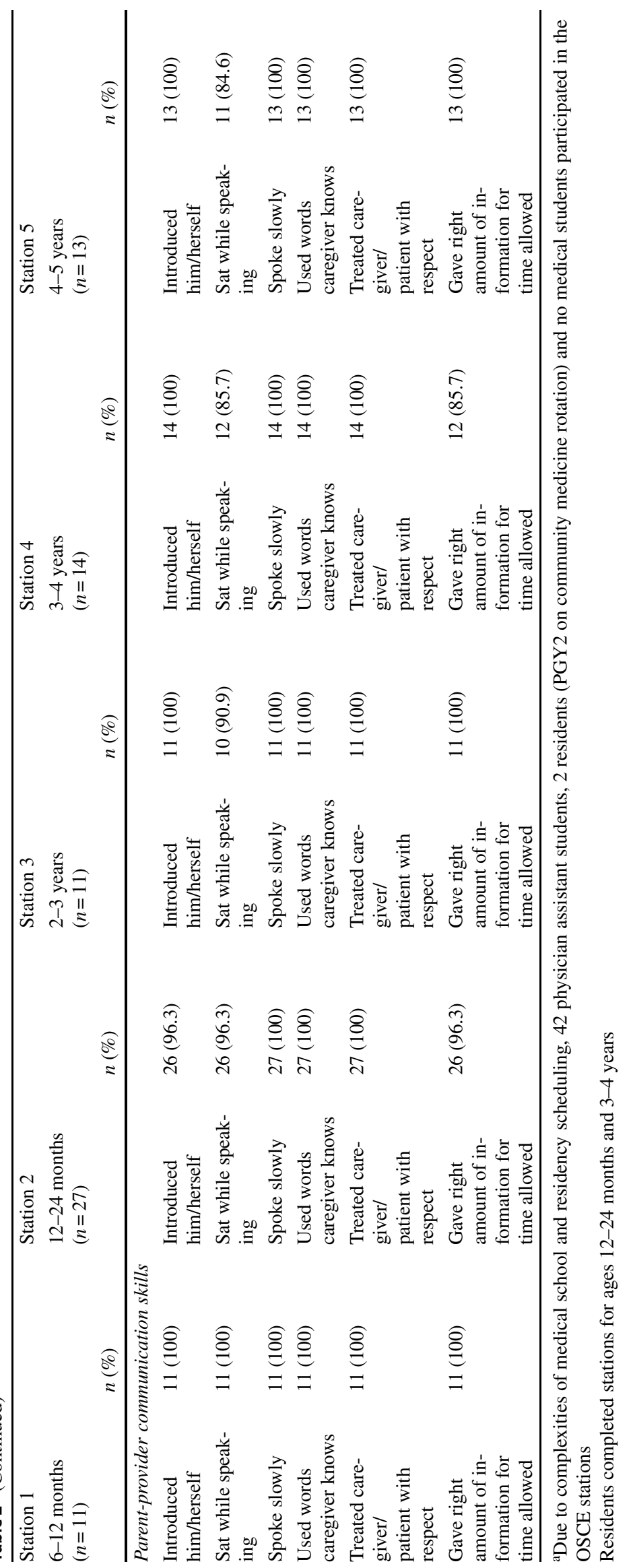


Read can make children more receptive to reading, more likely to read with their families, more likely to reach developmental language milestones, and make parents more compliant to well-child visits $[6,8,19]$. While these studies have demonstrated improvements in patient outcomes, little emphasis is placed on training of providers. With more simulated training of future providers, the impact of Reach Out and Read could be even greater.

Strengths of our curriculum include the use of interprofessional learners and trained standardized patients/ caregivers. A limitation was that not all learners completed all curriculum activities. Only $56.7 \%$ completed preand post-tests. However, this response rate is higher than previous studies evaluating online survey responses [20]. Due to the complexities of residency and medical school schedules, only two residents and no medical students were able to complete the OSCE and debriefing session. OSCE stations were held on one day. Future OSCEs should be designed to better suit residency and medical student schedules. Our OSCE stations were not specifically designed for homeless patients. The focus of our stations was on patients with minimal risk for language and developmental delays. This focus was due to learners' limited exposure to paediatric patients. Future OSCEs could be modified to address barriers experienced by underserved populations. While our curriculum was only evaluated at one institution, the supplementary materials provided can be tailored for other programs interested in paediatric literacy training, regardless of formal participation in Reach Out and Read.

Learners who participated in all or parts of our curriculum demonstrated significant improvements in knowledge and attitudes towards paediatric literacy concepts. While we experienced several logistical challenges, we were able to create a greater awareness of the importance of paediatric literacy in different phases of medical education and offer service learning in homeless shelter clinics participating in Reach Out and Read. Our next steps are to evaluate the curriculum impact on communication with and reading behaviours among mothers and children at the shelters and identify ways to empower future providers to participate in Reach Out and Read or similar programs in their practices.

Acknowledgements The authors would like to acknowledge the volunteers who served as standardized patients and standardized patient caregivers, the learners who completed curriculum elements and Jocelyn McConnell from Reach Out and Read Texas for her continued support of our research.

Funding This work was supported by the University of Texas Southwestern Academy of Teachers (SWAT) Junior Faculty Development Fund.

Conflict of interest T. Kindratt, B. Bernard, J. Webb and P. Pagels declare that they have no competing interests.
Ethical standards The University of Texas Southwestern Medical Center's Institutional Review Board deemed this project as non-human subjects research. No written informed consent was required. Verbal informed consent was obtained. All research project procedures were completed in accordance with the Declaration of Helsinki.

Open Access This article is distributed under the terms of the Creative Commons Attribution 4.0 International License (http:// creativecommons.org/licenses/by/4.0/), which permits unrestricted use, distribution, and reproduction in any medium, provided you give appropriate credit to the original author(s) and the source, provide a link to the Creative Commons license, and indicate if changes were made.

\section{References}

1. Jansen KL, Rosenbaum ME. The state of communication education in family medicine residencies. Fam Med. 2016;48:445-51.

2. Acceditation Council for Graduate Medical Education. ACGME common program requirements. 2017.

3. Accreditation Review Commission on Education for the Physician Assistant. Accreditation standards for physician assistant education. 2016.

4. American Academy of Family Physicians. Promoting early literacy development. 2017.

5. Hutton JS, Phelan K, Horowitz-Kraus T, et al. Shared reading quality and brain activation during story listening in preschool-age children. J Pediatr. 2017;191:204-211.e1.

6. Zuckerman B, Khandekar A. Reach out and read: evidence based approach to promoting early child development. Curr Opin Pediatr. 2010;22:539-44.

7. Klass P, Dreyer BP, Mendelsohn AL. Reach out and read: literacy promotion in pediatric primary care. Adv Pediatr. 2009;56:11-27.

8. Yaun J, Bach M, Bakke J, Goedecke PJ, Baldwin KM, Hare M. Evaluation of a language and literacy enhancement program. Clin Pediatr (phila). 2019;58:100-9.

9. Rosenthal MS, Werner MJ, Dubin NH. The effect of a literacy training program on family medicine residents. Fam Med. 2004;36:582-7.

10. Hazzard A, Dabrow S, Celano M, et al. Training residents in pediatric literacy: impact on knowledge, attitudes and practice. Ambul Child Health. 2000;6::237-46.

11. Turner JL, Dankoski ME. Objective structured clinical exams: a critical review. Fam Med. 2008;40:574-8.

12. Pagels P, Kindratt T, Arnold D, Brandt J, Woodfin G, Gimpel N. Health literacy objective structured clinical exam for family medicine residents. Med Teach. 2013;35:874-5.

13. Rodder SG, Kindratt TB, Xiao C, et al. Teaching and evaluating smartphone applications: the effectiveness of a curriculum expansion. Educ Health (Abingdon). 2018;31:95-102.

14. Gimpel N, Pagels P, Roy V, et al. Family medicine resident education: an innovative model of community medicine training. J Community Med Health Educ. 2013;3:1-5.

15. Kindratt T, Pagels P, Bernard B, et al. Teaching future primary care providers about parent-provider literacy communication. Educ Prim Care. 2018;29:250-1.

16. Reach Out and Read.. http://www.reachoutandread.org. Accessed 7 Nov 2018.

17. Kindratt T, Pagels $P$, Chung K, et al. Improving the Reach Out and Read program at a student-run free clinic for homeless women and children. J Stud Run Clin. 2017;3:1-8.

18. Frye AW, Hemmer PA. Program evaluation models and related theories: AMEE guide no. 67. Med Teach. 2012;34:e288-e99. 
19. Needlman RD, Dreyer BP, Klass P, Mendelsohn AL. Attendance at well-child visits after reach out and read. Clin Pediatr (Phila). 2019; https://doi.org/10.1177/0009922818822975.

20. Cook C, Health F, Thompson R. A meta-analysis of response rates in web- or internet-based survey. Educ Psychol Meas. 2000;60:821-36.

Tiffany Kindratt $\mathrm{PhD}, \mathrm{MPH}$, is the director of research in the Department of Physician Assistant Studies at UT Southwestern. Her research focuses on ways to reduce health disparities and improve communication between healthcare providers and their patients through medical education, community-based participatory research, and national survey methodologies.

Brittany Bernard MPAS, PA-C, is a paediatric trauma advanced practice provider at Children's Minnesota. She participated in this study as part of her graduate project for her Master of Physician Assistant Studies degree at the University of Texas Southwestern Medical Center in Dallas, Texas.
Jade Webb MPAS, PA-C, is a physician assistant practising in Dallas, Texas. She participated in this study as part of her graduate project for her Master of Physician Assistant Studies degree at the University of Texas Southwestern Medical Center in Dallas, Texas.

Patti Pagels MPAS, PA-C, has taught and developed curricula to address cultural competency and health literacy for medical learners nationally. Her interests include social determinants of health, health literacy, care of underserved communities and mentoring students as managers of student run clinics. She serves communities in Malawi, Africa and Guatemala. 